\title{
Non- Newtonian behavior of blood in very narrow vessels
}

\author{
Amit Kr. Chaubey, R. R. Yadav \\ SR Institute of Management \& Technology, Luck now
}

\begin{abstract}
Abstarct: The purpose of the study is to get some qualitative and quantitative insight into the problem of flow in vessels under consideration where the concentration of lubrication film of plasma is present between each red cells and tube wall. This film is potentially important in region to mass transfer and to hydraulic resistance, as well as to the relative resistance times of red cells and plasma in the vessels network.
\end{abstract}

\section{Introduction}

Prothero and Burton [10,11]. It is expected that the cells are deformed elastically to enable them to pass through the tube which also suffer some small elastic distension.. Prothero and Buston [10] pointed out that the bolus of viscous plasma between two cells must perform, relative to their motion, a toroidal circulation, towards on the tube axis and backward near the walls. Prothero and Buston estimated from their model experimental, the pressure drop in the bolus of moving plasma between two red cells and deduced a contribution to over all vessels resistance less than that given by Poiseuille law, mainly because plasma with depleted RBC has a viscosity considerably lower than typical values measured for whole blood.

A large number of theoretical and experimental efforts have been made in the literature to explain the blood flow behavior when it flows through the vessels of circulatory system of the living things. To account for the numerous relevant and important contribution of Bayliss[2], Womeresly[18,19], Whitemore [20], Attingn [1], Fung[5] and many others, mathematical modeling of blood flow has been subject to constant changes and modification. Above listed investigators have been used single - phase homogeneous Newtonian viscous fluid, a classical approach that does not account for the presence of red cells in blood while flowing the circulatory system. Although, this approach provides satisfactory tools to describe certain aspects of blood flow in aorta and large arteries, it fails to give an adequate representation of flow field, especially in the vessels of small diameter Srivastva and Srivastva[12-15] and Vann and Fitz-Gerald [17]. Several researcher Carmand Kurland[3],Gupta et al.[6], Chaturmani and Mahajan[4], Jean and Peddison[7], Jung and Cowokers[8,9] have pointed out that blood being a suspension of corpuscles, behave like a non-Newtonian fluid at low shear rates. Thurston [16] has developed a mathematical model for the flow of closely fitting incompressible elastic sphere in a tube under zero drag condition.

\section{Mathematical Analysis}

We consider the flow of elastic incompressible sphere in a rigid tube of uniform radius. Single file flow of RBC surrounded by an annulus of plasma is considered. In the case of movable buoyant particle treated in the present study, the condition of zero drag on the particle must be satisfied in addition to the Reynolds equation. It can be used to eliminate leak-back (which is equal to the discharge of the fluid observed relative to a reference frame fixed to the particle) leaving only pressure drop as an unknown.

The single RBC of biconcave - disk shape is deformed during the flow passage in very narrow vessels, as shown in figure.2.1.

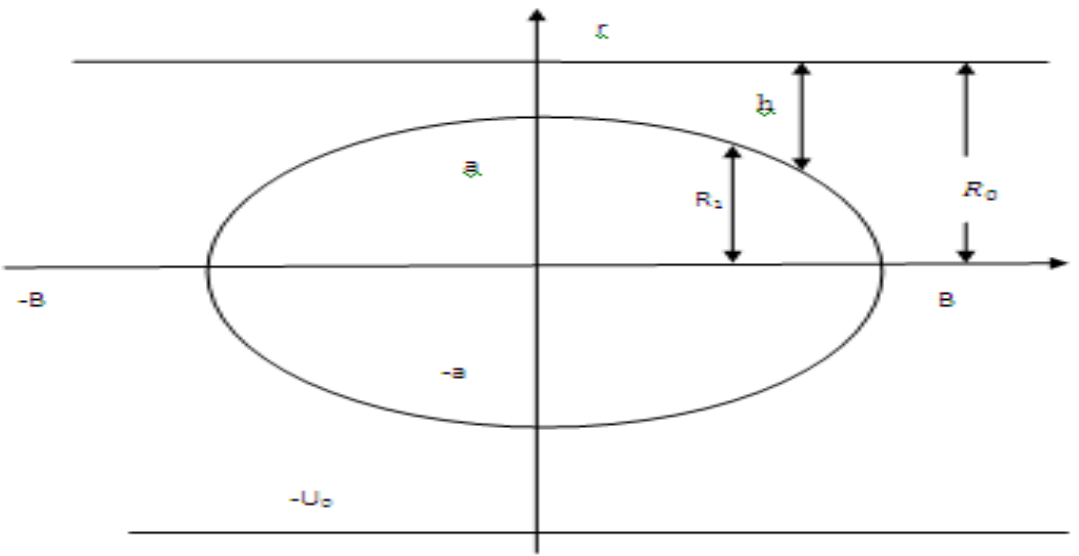

Figure.2.1 
It is assumed that the inertial terms are negligible, the equation of motion in cylindrical polar co-ordinate about the axis of symmetry is

$$
\frac{\partial p}{\partial z}=\frac{\mu}{r} \frac{\partial}{\partial r}\left(r \frac{\partial v_{z}}{\partial r}\right)
$$

The equation of continuity is

$$
\frac{\partial}{\partial r}\left(r v_{r}\right)+\frac{\partial}{\partial z}\left(r v_{z}\right)=0
$$

where $v_{r}$ and $v_{z}$ are the radial and axial velocity respectively, $\mu$ the dynamic viscosity of fluid, $p$ the pressure not varying with $r$.

Boundary conditions are

$$
\begin{array}{cc}
\mathrm{r}=\mathrm{R}_{0} ; \quad \mathrm{v}_{\mathrm{z}}=-\mathrm{v}_{0} ; & \mathrm{v}_{\mathrm{r}}=0 \\
\mathrm{r}=\mathrm{R}_{1} ; \quad \mathrm{v}_{\mathrm{z}}=0 ; & \mathrm{v}_{\mathrm{r}}=0 \\
\mathrm{P}(-\mathrm{b})-\mathrm{P}(\mathrm{b})=\Delta \mathrm{P}_{0} ; & \mathrm{h}=\mathrm{R}_{0}-\mathrm{R}_{1}
\end{array}
$$

For incompressible, continuity equation (2.2.2), we have

$$
\text { Or } \quad \begin{aligned}
-\int \frac{\partial}{\partial r}\left(r v_{r}\right) d r & =\int \frac{\partial}{\partial z}\left(r v_{z}\right) d r \\
\frac{\partial}{\partial z} \int_{R_{1}}^{R_{0}} r v_{z} d r & =-\int_{R_{1}}^{R_{0}} \frac{\partial}{\partial r}\left(r v_{r}\right) d r \\
& =0
\end{aligned}
$$

Further, $\int_{R_{1}}^{R_{0}} r v_{z} d r=C=-Q_{0}$

where $\mathrm{Q}_{0}$ is the leak- back, given by

$$
2 \pi R_{0} Q_{0}=\pi R_{0}^{2} U_{0}-\pi R_{0}^{2} V_{0}
$$

$\mathrm{V}_{0}$ is the average velocity of the fluid in lubrication zone.

From equation (2.2.7), we have

$$
Q_{0}=\frac{R_{0}}{2}\left(U_{0}-V_{0}\right)
$$

Integrating equation (2.2.1), we get

$$
v_{z}=\frac{1}{4 \mu} \frac{d p}{d z} r^{2}+A \log r+B
$$

where $\mathrm{A}$ and $\mathrm{B}$ are constant to be determine, by boundary condition (2.2.3) and (2.2.4) as

$$
\begin{aligned}
A & =\frac{-\left[U_{0}+\frac{1 d p}{4 \mu d z}\left(R_{0}^{2}-R_{1}^{2}\right)\right]}{\log \frac{R_{0}}{R_{1}}} \\
B & =\frac{\frac{1 d p}{4 \mu d z} R_{1}^{2}+\left[U_{0}+\frac{1 d p}{4 \mu d z}\left(R_{0}^{2}-R_{1}^{2}\right)\right] \log R_{1}}{\log \frac{R_{0}}{R_{1}}}
\end{aligned}
$$

and

Substituting the value of $\mathrm{A}$ and $\mathrm{B}$ in equation (2.2.9), we get

$$
v_{z}=\frac{1}{4 \mu} \frac{d p}{d z}\left[r^{2}-R_{1}^{2}+\frac{\left(R_{0}^{2}-R_{1}^{2}\right)}{\log \frac{R_{1}}{R_{0}}} \log \frac{r}{R_{1}}+U_{0} \frac{\log \frac{r}{R_{1}}}{\log \frac{R_{1}}{R_{0}}}\right]
$$

Put $R_{0}=R_{1}+h$ in equation (2.2.10) becomes

$$
v_{z}=\frac{1}{4 \mu} \frac{d p}{d z}\left[r^{2}-R_{1}^{2}+\frac{\left(2 R_{1} h+h^{2}\right)}{\log \left(1+\frac{h}{R_{1}}\right)} \log \frac{r}{R_{1}}+U_{0} \frac{\log \frac{r}{R_{1}}}{\log \left(1+\frac{h}{R_{1}}\right)}\right]
$$

Integrating equation (2.2.11) and using (2.2.6), we have

$$
\frac{d p}{d z}=\frac{16 \mu\left[R_{0} Q_{0}-U_{0}\left(\frac{R_{0}^{2}}{2}+\frac{\left(R_{0}^{2}-R_{1}^{2}\right)}{\left.4 \log \frac{R_{1}}{R_{0}}\right)}\right)\right]}{\left(R_{0}^{2}-R_{1}^{2}\right)\left[\left(R_{0}^{2}+R_{1+}^{2} \frac{\left(R_{0}^{2}-R_{1}^{2}\right)}{\log \frac{R_{1}}{R_{0}}}\right)\right]}
$$

Under zero - drag condition, we have

Pressure force acting on the particle + viscous stresses experienced by the particle $=0$.

i.e. $\quad \pi \int_{-b}^{b} R_{1}^{2} \frac{d p}{d z} d z-2 \pi \mu \int_{-b}^{b} R_{1}\left(\frac{\partial v_{z}}{\partial r}\right)_{r=R_{1}} d z=0$

If $\Omega$ is fluid volume, then from equation (2.2.1), we have

$$
\begin{aligned}
& \int_{\Omega} \frac{d p}{d z} \cdot d \Omega=\mu \int_{\Omega} \frac{1}{\mathrm{r}} \frac{\partial}{\partial r}\left(r \frac{\partial v_{z}}{\partial r}\right) d \Omega \\
& \frac{1}{\mu}\left\{\pi R_{0}^{2}[\mathrm{P}(\mathrm{b})-\mathrm{P}(-\mathrm{b})]-\pi \int_{-b}^{b} R_{1}^{2} \frac{d p}{d z} d z\right\} \\
& =2 \pi R_{0} \int_{-b}^{b}\left(\frac{\partial v_{z}}{\partial r}\right)_{r=R_{0}} d z-2 \pi \int_{-b}^{b} R_{1}\left(\frac{\partial v_{z}}{\partial r}\right)_{r=R_{1}} d z
\end{aligned}
$$

From equation (2.2.13) and equation (2.2.15), we have

$$
\pi R_{0}^{2} \Delta \mathrm{P}_{0}=-2 \pi \mu R_{0} \int_{-b}^{b}\left(\frac{\partial v_{z}}{\partial r}\right)_{r=R_{0}} d z
$$

Using the quantity $\mu U / a$, in pressure and stress terms, the non dimensional quantities becomes, 
$b^{\prime}=\frac{p a}{\mu U}, \tau^{\prime}=\frac{\tau a}{\mu U}, U_{0}{ }^{\prime}=\frac{U_{0}}{U}, v_{z}{ }^{\prime}=\frac{v_{z}}{U}, z^{\prime}=\frac{z}{R_{0}}, r^{\prime}=\frac{r}{a}$,

$R_{1}{ }^{\prime}=\frac{R_{1}}{R_{0}}, \mathrm{H}=\frac{h}{R_{0}}, \alpha=\frac{a}{b}, \beta=\frac{a}{R_{0}}, C_{0}=\frac{2 Q_{0}}{U R_{0}}$,

Velocity field $\mathrm{v}_{\mathrm{z}}$ and pressure gradient $\frac{d p}{d z}$ given by equation (2.2.11) and (2.2.12) take the form,

$$
\begin{aligned}
& v_{z}^{\prime}\left(r^{\prime}, z^{\prime}\right)= \frac{1}{4 \beta} \frac{d p^{\prime}}{d z^{\prime}}\left[\beta^{2} r^{\prime 2}-R_{1}^{\prime 2}+\frac{1-R_{1}^{2}}{\log R_{1}^{\prime}} \log \frac{\beta r^{\prime}}{R_{1}^{\prime}}\right] \\
&+U_{0}^{\prime} \frac{\log \frac{\beta r^{\prime}}{R_{1}^{\prime}}}{\log R_{1}^{\prime}} \\
& \frac{d p^{\prime}}{d z}=8 \beta \frac{\left[C_{0}-U_{0}\left(1+\frac{1-R_{1}^{2}}{\left.2 \log R_{1}^{\prime}\right)}\right)\right]}{\left(1-R_{1}^{2}\right)\left[\left(1+R_{1}^{2}+\frac{\left(1-R_{1}^{2}\right)}{\log R_{1}}\right)\right]}
\end{aligned}
$$

For the shake of convenience, we omit " in proceeding expression.

Equation (2.2.18) gives

$$
\frac{\partial V_{z}}{\partial r}=\frac{1}{4 \beta} \frac{d p}{d z}\left[2 \beta^{2} r+\frac{1-R_{1}^{2}}{\log R_{1}}\right]+\frac{U_{0}}{2 \log R_{1}}
$$

With the help of equation (2.2.20) and (2.2.16), we obtain

If we put

$$
\Delta \mathrm{P}_{0}=4 \beta \int_{-}^{\beta} \beta / \alpha\left[\frac{\left\{2+\frac{1-R_{1}^{2}}{\log R_{1}}\right\}\left\{C_{0}-U_{0}\left(1+\frac{1-R_{1}^{2}}{2 \log R_{1}}\right)\right\}}{\left(1-R_{1}^{2}\right)\left\{1+R_{1}^{2}+\frac{\left(1-R_{1}^{2}\right)}{\log R_{1}}\right\}}+\frac{U_{0}}{2 \log R_{1}}\right] d z
$$

As

$$
\Delta \mathrm{P}_{0}=\mathrm{P}\left(-\frac{\beta}{\alpha}\right)-\mathrm{P}\left(\frac{\beta}{\alpha}\right)
$$

Then

$$
\Delta \mathrm{P}_{0}=8 \beta \int_{-\beta}^{\beta / \alpha} / \alpha \frac{\left\{C_{0}-U_{0}\left(1+\frac{1-R_{1}^{2}}{2 \log R_{1}}\right)\right\}}{\left(1-R_{1}^{2}\right)\left\{1+R_{1}^{2}+\frac{\left(1-R_{1}^{2}\right)}{\log R_{1}}\right\}} d z
$$

$$
\begin{aligned}
& D_{11}=4 \beta \int_{-}^{\beta / \alpha} / \alpha\left[\frac{\left\{2+\frac{1-R_{1}^{2}}{\log R_{1}}\right\}}{\left(1-R_{1}^{2}\right)\left\{1+R_{1}^{2} \frac{\left(1-R_{1}^{2}\right)}{\log R_{1}}\right\}}\right] d z \\
& D_{12}=4 \beta \int_{-}^{\beta / \alpha} / \alpha\left[\frac{\left\{2+\frac{\left.1-R_{1}^{2}\right)}{\log R_{1}}\right)\left\{\left(1+\frac{\left(1-R_{1}^{2}\right)}{2 \log R_{1}}\right\}\right.}{\left(1-R_{1}^{2}\right)\left\{1+R_{1}^{2}+\frac{\left(1-R_{1}^{2}\right)}{\log R_{1}}\right\}}-\frac{1}{2 \log \mathrm{R}_{1}}\right] d z \\
& D_{21}=8 \beta \int_{-}^{\beta} / \alpha \frac{d z}{\left(1-R_{1}^{2}\right)\left\{1+R_{\left.1+\frac{\left(1-R_{1}^{2}\right)}{\log R_{1}}\right\}}\right.} \\
& D_{21}=8 \beta \int_{-}^{\beta} \beta / \alpha \frac{\left\{1+\frac{\left(1-R_{1}^{2}\right)}{2 \log R_{1}}\right\}}{\left(1-R_{1}^{2}\right)\left\{1+R_{1}^{2}+\frac{\left(1-R_{1}^{2}\right)}{\log R_{1}}\right\}} d z
\end{aligned}
$$

Then equation (2.2.21) takes the form

$$
\begin{aligned}
& D_{11} C_{0}+\Delta P_{0}=D_{12} U_{0} \\
& D_{21} C_{0}+\Delta P_{0}=D_{22} U_{0}
\end{aligned}
$$

For $\mathrm{U}_{0}=1$, above equation gives,

$$
\begin{aligned}
& C_{0}=\frac{D_{12}-D_{22}}{D_{11}-D_{21}}, \quad \Delta P_{0}=D_{12}-D_{11} C_{0} \\
& \frac{U_{0}}{V_{0}}=\left(1-C_{0}\right)^{-1} ;
\end{aligned}
$$

Effective viscosity

$$
\eta=\frac{\alpha U_{0} \Delta P_{0}}{16 V_{0}}
$$

\section{Result and Discussion}

We have calculated the value of $\frac{U_{0}}{V_{0}}$ and $\eta$ and compared the calculated value from the results obtained by other authors. The results are given in the form of tables.

Variation of velocity field $\mathrm{v}_{\mathrm{z}}$ can be obtained from equation (2.2.18). For different values of $\alpha(=1.5,1.0,0.5)$ and $\beta(=0.90,0.95,0.99)$, the variation of $\mathrm{v}_{\mathrm{z}}$ with respect to gap thickness $\mathrm{H}$ have been shown in the table $\mathrm{s} 2.1$ and 2.2. 
Table - 2.1

\begin{tabular}{|l|c|c|c|c|c|c|}
\hline $\begin{array}{l}\text { S. } \\
\text { No }\end{array}$ & $\begin{array}{c}\text { Shape of } \\
\text { the particle }\end{array}$ & $\beta$ & $\alpha$ & \multicolumn{2}{|c|}{$\begin{array}{c}\text { Value of } \eta \\
\text { obtained } \\
\text { by other authors }\end{array}$} & \multirow{2}{*}{$\begin{array}{c}\text { Value of } \eta \\
\text { obtained } \\
\text { in the present } \\
\text { analysis }\end{array}$} \\
\cline { 5 - 6 } & & & & & & \\
\hline 1 & Sphere & 0.70 & 1.0 & 1.196 & 1.196 & 0.853 \\
\hline 2 & Sphere & 0.80 & 1.0 & 1.455 & 1.455 & 1.177 \\
\hline 3 & Sphere & 0.90 & 1.0 & 2.201 & 2.200 & 1.971 \\
\hline 4 & Sphere & 0.93 & 1.0 & 2.813 & 2.611 & 2.540 \\
\hline 5 & Sphere & 0.95 & 1.0 & 3.485 & 3.259 & 3.207 \\
\hline 6 & Sphere & 0.98 & 1.0 & 6.207 & 5.870 & 5.853 \\
\hline 7 & Sphere & 0.99 & 1.0 & 9.274 & 9.013 & 8.964 \\
\hline 8 & Ellipsoid & 0.90 & 4.0 & 1.503 & 1.268 & 1.971 \\
\hline 9 & Ellipsoid & 0.90 & 0.5 & 1.795 & 1.714 & 1.971 \\
\hline
\end{tabular}

Table - 2.3.2

\begin{tabular}{|l|c|c|c|c|c|c|}
\hline $\begin{array}{l}\text { S. } \\
\text { No. }\end{array}$ & $\begin{array}{c}\text { Shape of the } \\
\text { particle }\end{array}$ & $\beta$ & $\alpha$ & \multicolumn{2}{|c|}{$\begin{array}{c}\text { Value of } \frac{U_{0}}{V_{0}} \text { obtained } \\
\text { by other authors }\end{array}$} & $\begin{array}{c}\text { Value of } \frac{U_{0}}{V_{0}} \\
\text { obtained } \\
\text { in the present } \\
\text { analysis }\end{array}$ \\
\cline { 5 - 6 } & & & {$[17]$} & {$[20]$} & 1.403 \\
\hline 1 & Sphere & 0.70 & 1.0 & 1.416 & 1.414 & 1.268 \\
\hline 2 & Sphere & 0.80 & 1.0 & 1.272 & 1.274 & 1.133 \\
\hline 3 & Sphere & 0.90 & 1.0 & 1.136 & 1.132 & 1.092 \\
\hline 4 & Sphere & 0.93 & 1.0 & 1.092 & 1.092 & 1.066 \\
\hline 5 & Sphere & 0.95 & 1.0 & 1.068 & 1.066 & 1.027 \\
\hline 6 & Sphere & 0.98 & 1.0 & 1.029 & 1.026 & 1.013 \\
\hline 7 & Sphere & 0.99 & 1.0 & 1.019 & 1.103 & 1.133 \\
\hline 8 & Ellipsoid & 0.90 & 4.0 & 1.138 & 1.132 & \\
\hline 9 & Ellipsoid & 0.90 & 0.5 & 1.136 & 1.132 & 1.133 \\
\hline
\end{tabular}

\section{References}

[1]. Attinger, E. O. : Pulsatile blood flow , McGraw-Hill. New-York (1964).

[2]. Baylish,L .E. : Rheology of blood and hymph , In: Deformation of flow in Biological system . A. Fry-Wssyling (Ed.)North Holland Pub. Co. Amsterdom (1952).

[3]. Charms, S.E. and G. S. Kurland : Blood flow and microcirculation, Willy, New york,(1974).

[4]. Chaturani, P. and S. P. Mahajan : Poiseuille flow of micropolar fluid with non -zero couple stress at boundary with application to blood, Biorheology 19(1982),pp.507-518.

[5]. Fung, Y. C. : Biomechanics, its scope, history and some problem of continuum mechanics in Phsiology, Appl. Mech. Riew 21(1964), pp.1-20.

[6]. Gupta, B. B., K. M. Nigam and M. Y. Jaffrin : A three layer semi- empirical model for blood flow and particular suspension through narrow tubes. ASME, J. of Biomechanical Eng.104(1982),507-518.

[7]. Jean, T.H. and J. Peddison : Mathematical modeling of particular suspension in vertical circular pipe flow. Int. J. of Eng. Sci. 39(2001), pp.1167-1189.

[8]. Jung, J., A. Hassanein, and R.W. Lyczkowashi : Hemodynamics computation using multiphase flow dynamics in right coronary artery, Annls. Of Biomechanical Eng . 34(2006a), pp.393-402.

[9]. Jung, J., R.W. Lyczkowashi, C. B. Panchal A. Hassanein : Hemodynamics simulation of pulsatile flow in a coronary artery , Annls. Of Biomechanical Eng. 39(2006b),pp.2064-2073.

[10]. Prothero, J. and A.C. Burton : The physics of blood flow in capillaries I., 'The nature of motion' ,J. of Biophysics,1(1961), pp.565-579.

[11]. Prothero, J. and A.C. Burton : The physics of blood flow in capillariesII. 'The capillary resistence to flow', J. of Biophysics 2(1962),pp.199-212.

[12]. Srivastva, L. M. and V.P. Srivastva : On two phase model of pulsatile blood flow with entrance effect , Biorheology 20(1983), pp.761-777.

[13]. Srivastva, L. M., Edemeka, U. E. and V.P. Srivastva : Particular Suspension blood flow under external body acceleration, Int. J. of Biomedical computing 37(1994),113-129.

[14]. Srivastva, V.P. : Blood flow through stenotic vessels with a peripheral plasma layer and application . Automedica 18(2000), pp.271300 .

[15]. Srivastva, V.P. : Flow of a couple stress fluid representing blood flow through steno tic vessels with a peripheral layer . Int. J. of Pure and Appl. Mathematics. 34(2003), pp .1727-1740. 
[16]. Thurston, G. B. : Plasma release - cell layering theory for blood flow . Biorheology , 26(1989),pp.199-214

[17]. ann, P.G. and J. M. Fitz-Gerald: Flow mechanics of red cells train in very narrow capilary - I. Train of uniform cells. Micro vascular Research, 24 (1982), pp.296-313.

[18]. Womersely, J. R.: Oscillatory motion of a viscous liquid in a thin walled elastic tube $-\mathrm{I}$. The linear approximation for long wave . Philosphical Magezine, 46(1955), pp.199-221.

[19]. Womersely, J. R. : An elastic theory of pulse transmission and oscillatory flow in mammalian artries, WADCreport TR, (1957)pp. 56-614.

[20]. Whitmore, R. L.: A theory of blood flow in small vessels, J. of Appl. Physiology, 20(1967), pp.767-771. 